\title{
The role of the Acanthamoeba castellanii Sir2-like protein in the growth and encystation of Acanthamoeba
}

\author{
So-Young Joo' ${ }^{1}$, Ja Moon Aung ${ }^{1}$, Minsang Shin'², Eun-Kyung Moon ${ }^{3}$, Hyun-Hee Kong ${ }^{4}$, Youn-Kyoung Goo', \\ Dong-ll Chung ${ }^{1}$ and Yeonchul Hong ${ }^{1 *}$
}

\begin{abstract}
Background: The encystation of Acanthamoeba leads to the development of resilient cysts from vegetative trophozoites. This process is essential for the survival of parasites under unfavorable conditions. Previous studies have reported that, during the encystation of $A$. castellanii, the expression levels of encystation-related factors are upregulated. However, the regulatory mechanisms for their expression during the encystation process remains unknown. Proteins in the sirtuin family, which consists of nicotinamide adenine dinucleotide-dependent deacetylases, are known to play an important role in various cellular functions. In the present study, we identified the Acanthamoeba silent-information regulator 2-like protein (AcSir2) and examined its role in the growth and encystation of Acanthamoeba.

Methods: We obtained the full-length sequence for AcSir2 using reverse-transcription polymerase chain reaction. In Acanthamoeba transfectants that constitutively overexpress AcSir2 protein, SIRT deacetylase activity was measured, and the intracellular localization of AcSir2 and the effects on the growth and encystation of trophozoites were examined. In addition, the sirtuin inhibitor salermide was used to determine whether these effects were caused by AcSir2 overexpression
\end{abstract}

Results: AcSir2 was classified as a class-IV sirtuin. AcSir2 exhibited functional SIRT deacetylase activity, localized mainly in the nucleus, and its transcription was upregulated during encystation. In trophozoites, AcSir2 overexpression led to greater cell growth, and this growth was inhibited by treatment with salermide, a sirtuin inhibitor. When AcSir2 was overexpressed in the cysts, the encystation rate was significantly higher; this was also reversed with salermide treatment. In AcSir2-overexpressing encysting cells, the transcription of cellulose synthase was highly upregulated compared with that of control cells, and this upregulation was abolished with salermide treatment. Transmission electron microscope-based ultrastructural analysis of salermide-treated encysting cells showed that the structure of the exocyst wall and intercyst space was impaired and that the endocyst wall had not formed.

Conclusions: These results indicate that AcSir2 is a SIRT deacetylase that plays an essential role as a regulator of a variety of cellular processes and that the regulation of AcSir2 expression is important for the growth and encystation of A. castellanii.

Keywords: Acanthamoeba castellanii, Encystation, Sirtuin-like protein, Salermide

${ }^{*}$ Correspondence: ychong@knu.ac.kr

${ }^{1}$ Department of Parasitology and Tropical Medicine, School of Medicine, Kyungpook National University, Daegu, Republic of Korea

Full list of author information is available at the end of the article

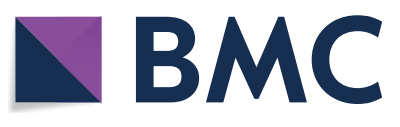

(c) The Author(s) 2020. This article is licensed under a Creative Commons Attribution 4.0 International License, which permits use, sharing, adaptation, distribution and reproduction in any medium or format, as long as you give appropriate credit to the original author(s) and the source, provide a link to the Creative Commons licence, and indicate if changes were made. The images or other third party material in this article are included in the article's Creative Commons licence, unless indicated otherwise in a credit line to the material. If material is not included in the article's Creative Commons licence and your intended use is not permitted by statutory regulation or exceeds the permitted use, you will need to obtain permission directly from the copyright holder. To view a copy of this licence, visit http://creativeco mmons.org/licenses/by/4.0/. The Creative Commons Public Domain Dedication waiver (http://creativecommons.org/publicdomain/ zero/1.0/) applies to the data made available in this article, unless otherwise stated in a credit line to the data. 


\section{Background}

Acanthamoeba, an opportunistic human pathogen that causes granulomatous amoebic encephalitis, dermatitis, and amoebic keratitis, is widely distributed in both natural and artificial environments (see $[1,2]$ for a review). The life-cycle of Acanthamoeba has both trophozoites and cysts stages. Under conditions that are unfavorable for proliferation, such as a lack of nutrients, low temperatures, low moisture levels, conditions leading to hyperosmolarity, and the presence of biocides, vegetative Acanthamoeba trophozoites become metabolically inactive, dormant cysts via encystation [3-6]. Acanthamoeba cysts are double-walled and highly resistant to adverse conditions, thus prolonging their survival and facilitating their transmission [1,3-8]. Thus, the inhibition of encystation during the treatment of amoebic infections can lead to more favorable outcomes. During the encystation process of Acanthamoeba, many encystation-related proteins, including cyst-specific protein (CSP21) [9], various proteases [10-13], autophagy-related factors [14-16], and cellulose synthase [17-20], have been shown to be upregulated and to play an important role in cyst formation. However, how the expression of encystation-related factors is controlled during the encystation process remains unclear.

Previously, Kong et al. [21] investigated encystationmediating factors using the comparative microarray analysis of trophozoites and cysts. Using data mining of the DNA sequences of the upregulated genes in the encysting trophozoites, a cDNA fragment containing a member of the sirtuin family of proteins, which are transcription regulators, was found. Sirtuins are a silent-information regulator 2 (SIR2)-like family of protein deacetylases that require nicotinamide adenine dinucleotide (NAD) as a cofactor in the deacetylation reaction. Thus, it is generally believed that sirtuins act as a sensor of energetic status that interprets external changes and regulates internal responses at a subcellular level [22-24]. Sirtuins have been evolutionarily conserved from bacteria to humans. In humans, seven sirtuin family members (SIRT1 to SIRT7) have been reported, with each differing in terms of their subcellular localization and protein substrates [25]. SIRT1, SIRT6, and SIRT7 are found primarily in the nucleus, SIRT2 is localized in the cytosol, and SIRT3, SIRT4, and SIRTt5 are mitochondrial proteins. Given that they are found in different locations within a cell, sirtuins are involved a variety of critical cellular processes, including transcription, DNA repair, metabolism, and stress resistance $[26,27]$. Additionally, sirtuins regulate the number, activity, and turnover of mitochondria [28].

Sirtuins have been positively associated with a longer life span in some organisms and the potential regulation of pathways mediated by nutrient starvation (see [29,30] for a review). They have also been connected to numerous human diseases [31], thus sirtuin inhibitors have attracted significant attention as potential therapeutics [32]. In some parasites, sirtuins play a crucial role in the modulation of parasite gene expression, and a significant number are known to be crucial to the survival and/or development of several parasites under various conditions; as a result, this family of proteins has been identified as a potential target for the development of anti-parasitic therapies [33, 34]. For example, the sirtuin inhibitors sirtinol and salermide have been shown to interfere with the growth of Leishmania infantum [35] and Trypanosoma cruzi [36], respectively, while, in Schistosoma mansoni, some sirtuin inhibitors induce the apoptosis and death of schistosomula, the disruption of adult worm pairs, and a reduction in egg-laying [37, 38].

In the present study, we identify a sirtuin in $A$. castellanii, examine its role in trophozoite growth and encystation, and assess its potential as a therapeutic target in the treatment of Acanthamoeba infections.

\section{Methods \\ Acanthamoeba castellanii cultures and the induction of encystation}

The Neff strain of Acanthamoeba castellanii (ATCC \#30010) was obtained from the American Type Culture Collection (Manassas, VA, USA) and cultured in peptone-yeast-glucose (PYG) medium at $25^{\circ} \mathrm{C}$, as previously described [39]. The encystation of Acanthamoeba was then induced as previously described with slight modifications [40]. Briefly, $1 \times 10^{6} \mathrm{~A}$. castellanii cells in their postlogarithmic growth phase were collected and washed with phosphate-buffered saline (PBS) and incubated in $10 \mathrm{ml}$ of encystment medium $(95 \mathrm{mM} \mathrm{NaCl}, 5 \mathrm{mM} \mathrm{KCl}, 8 \mathrm{mM}$ $\mathrm{MgSO}_{4}, 0.4 \mathrm{mM} \mathrm{CaCl}_{2}, 1 \mathrm{mM} \mathrm{NaHCO}_{3}$ and $20 \mathrm{mM}$ Tris$\mathrm{HCl}, \mathrm{pH}$ 9.0) at $25^{\circ} \mathrm{C}$ for $72 \mathrm{~h}$. The morphological transformation of the cells into cysts was observed using light microscopy. The number of cells was counted after treating the cells with $0.4 \%$ trypan blue, which selectively stains non-viable cells [41]. Encystation efficiency was assessed by counting the number of cysts under a light microscope after treating the cells with $0.5 \%$ sodium dodecyl sulfate [42].

\section{Cloning, quantitative transcriptional profiling and phylogenetic analysis of AcSir2}

The full-length cDNA sequence for AcSir 2 was isolated from AmoebaDB (AmoebaDB: ACA1_084880) [43] and verified using reverse transcription polymerase chain reaction (RT-PCR) using sequence-specific primers (sense: $5^{\prime}$-ATG GCC AGC ACA GTC GAC TC-3'; antisense: 5'-TTA GGC 
GAC GTC GAT CAG TT-3'). The expression of AcSir2 in both trophozoites and encysting cells were determined using quantitative real-time PCR (qRT-PCR). The total RNA of the Acanthamoeba trophozoites and cysts was purified using RNeasy Mini Kits following the manufacturer's instructions (Qiagen, Hilden, Germany). qRT-PCR was conducted using an Eco Real-Time PCR system (Illumina, SD, USA), as previously described [11]: $10 \mathrm{~min}$ of pre-incubation at $95^{\circ} \mathrm{C}$; followed by 40 cycles of $15 \mathrm{~s}$ at $95^{\circ} \mathrm{C}$ and $1 \mathrm{~min}$ at $60{ }^{\circ} \mathrm{C}$ with an AcSir2-specific primer (sense: $5^{\prime}$-CAC CTA CGA CCT CCA TCC GA-3'; antisense: $5^{\prime}$-CTT CTT CCA CTG GAC GGT GAC-3'). Relative amounts were calculated and normalized with respect to the level of Acanthamoeba actin as an internal standard (GenBank: CAA23399) [44] (sense primer 5'-AGG TCA TCA CCA TCG GTA ACG-3' and antisense primer $5^{\prime}$-TCG CAC TTC ATG ATC GAG TTG- $3^{\prime}$ ). The amino acid sequences were aligned using ClustalW and a phylogenetic tree was constructed using Geneious prime (Biomatters, http://www.geneious.com). Bootstrap proportions were used to assess the robustness of the tree with 1000 bootstrap replications.

\section{Overexpression of AcSir2 in A. castellanii}

The AcSir2 gene was cloned into a pGAPDH vector upstream of the enhanced green fluorescent protein (EGFP) reporter gene including an Acanthamoeba glyceraldehyde 3-phosphate dehydrogenase (GAPDH) promoter [45]. For transfection into Acanthamoeba, A. castellanii grown to the mid-log phase were washed with PBS and resuspended in $3 \mathrm{ml}$ of PYG culture medium. Twenty-four hours before transfection, $4 \times 10^{5}$ cells were added to each well of a 6 -well culture plate in $3 \mathrm{ml}$ of PYG medium and incubated at $25{ }^{\circ} \mathrm{C}$. Four micrograms of the cloned plasmid (pGAPDH-AcSir2-EGFP) was added to the PYG medium (total $100 \mu \mathrm{l}$ ) and mixed with $20 \mu \mathrm{l}$ of SuperFect transfection reagent (Qiagen). The mixture was then vortexed for $10 \mathrm{~s}$ and incubated for $10 \mathrm{~min}$ at room temperature to allow for the formation of the transfection complex. The medium was gently aspirated from the 6-well plate, and the amoeba cells were washed once with $3 \mathrm{ml}$ of PBS. After incubating the DNA with transfection reagent, $1 \mathrm{ml}$ of PYG medium was added to a reaction tube containing the transfection complex and mixed via pipetting. The mixture was then immediately transferred to the cells on the 6-well plate. The cells were incubated for $2-3 \mathrm{~h}$ at $25^{\circ} \mathrm{C}$, the medium removed, and the cells washed once with PBS before being allowed to recover in $3 \mathrm{ml}$ of PYG medium for $24 \mathrm{~h}$ at $25^{\circ} \mathrm{C}$. The transfected cells were then transferred to a selection medium containing $50 \mu \mathrm{g} / \mathrm{ml}$ of G418 for 2-3 weeks until cell growth was apparent. The overexpression of AcSir2-EGFP was assessed using qRT-PCR.

\section{Confocal microscopy and the determination of the SIRT deacetylase activity of AcSir2}

The amoebae expressing EGFP-fused AcSir2 were observed using an LSM 5 Exciter scalable confocal microscope (Zeiss, Hamburg, Germany). EGFP- or DAPI (4',6-diamidino-2-phenylindole)-mediated fluorescence was performed using band-pass filters at the excitation and emission wavelengths of 500-530 and 360-460 nm, respectively. Images were acquired and analyzed using a Zeiss LSM Image Examiner. SIRT deacetylase activity was measured with a Universal SIRT Activity Colorimetric Assay Kit (Abcam, Cambridge, MA, USA) according to the manufacturer's instructions. Briefly, nuclear extracts of the vector control and AcSir2-overexpressing trophozoites were prepared using a Nuclear Extract Kit (Thermo Fisher Scientific, Rockford, IL, USA) according to the manufacturer's recommendations. Nuclear extracts from each sample were incubated with the deacetylase substrate for $1 \mathrm{~h}$ at $37^{\circ} \mathrm{C}$ and then incubated sequentially with capture and detection antibodies, followed by the colorimetric reaction. Absorbance at 450 $\mathrm{nm}$ was measured with a reference absorbance at 655 $\mathrm{nm}$ using a microplate reader (Molecular Devices, Sunnydale, CA, USA).

\section{Transmission electron microscopy (TEM)}

Acanthamoeba castellanii trophozoites and cysts overexpressing EGFP and AcSir2-EGFP, respectively, were prepared for transmission electron microscopy (TEM) with or without the sirtuin inhibitor salermide as previously described, with slight modifications [11]. Briefly, suspensions of the trophozoites and cysts were centrifuged, and the obtained sediment washed 3 times in cold $1 \times$ PBS. The cells were prefixed using $2.5 \%$ glutaraldehyde in 0.1 $\mathrm{M}$ phosphate buffer ( $\mathrm{pH}$ 7.4) for $3 \mathrm{~h}$, rinsed with $0.1 \mathrm{M}$ phosphate buffer, post-fixed with $1 \%$ osmium tetroxide for $2 \mathrm{~h}$, and rinsed twice with $0.1 \mathrm{M}$ phosphate buffer. The fixed cells were then dehydrated using an ethyl alcohol gradient (50, 70, 80, 95 and 100\%), treated twice with propylene oxide resin (1:1) for $20 \mathrm{~min}$, and incubated in propylene oxide resin (1:1) overnight under continuous rotation. The cells were then embedded in epoxy resin (Embed-812; Electron Microscopy Sciences, PA, USA) and incubated at $37{ }^{\circ} \mathrm{C}$ for $12 \mathrm{~h}, 45^{\circ} \mathrm{C}$ for $12 \mathrm{~h}$, and $60{ }^{\circ} \mathrm{C}$ for $24 \mathrm{~h}$. Ultrathin sections were taken using a ReichertJung ultramicrotome and stained with uranyl acetate and lead citrate. The sections were observed using a transmission electron microscope (Hitachi H-7000, Tokyo, Japan).

\section{Flow cytometry}

DNA content and cell size were determined using flow cytometry (FACS Calibur; Becton Dickinson, Franklin Lakes, NJ, USA) and Cell Quest software. For the 
trophozoite cell size experiments, cells transfected with either the pGAPDH-EGFP or pGAPDH-AcSir2-EGFP plasmid and selected using G418 sulfate $(50 \mu \mathrm{g} / \mathrm{ml}$; Calbiochem, Darmstadt, Germany) were harvested for flow cytometry. To analyze the population of transfected cells, 10,000 EGFP-positive single cells were collected. The mean forward scatter height (FSC-H), which is correlated with cell size, and the optical refraction index of the outer membrane of the cell were determined as a measure of relative cell size [46]. To determine the DNA content, the cells were fixed with $3.7 \%$ formaldehyde, permeabilized with ethanol (at a final concentration of $70 \%$ ) overnight at $4{ }^{\circ} \mathrm{C}$, and then incubated in propidium iodide/RNase A solution $(10 \mu \mathrm{g} / \mathrm{ml}$ propidium iodide containing 250 $\mu \mathrm{g} / \mathrm{ml} \mathrm{RNase} \mathrm{A)} \mathrm{for} 15 \mathrm{~min}$ at $37^{\circ} \mathrm{C}$, followed by FACS analysis. FL2-A values correlate with the DNA content.

\section{Proliferation and encystation assays}

To determine the effect of the sirtuin inhibitor salermide (N-\{3-[(2-hydroxy-1-naphthalenylmethylene)-amino]phenyl\}-2-phenyl-propionamide; Sigma-Aldrich, MO, USA) on the proliferation and encystation of $A$. castellanii, $3 \times 10^{5}$ cells/well of $A$. castellanii trophozoites were seeded into 6-well plates containing $3 \mathrm{ml}$ of encystment medium per well. Subsequently, the trophozoites were treated with the inhibitor and incubated at $25^{\circ} \mathrm{C}$ for 24 and $48 \mathrm{~h}$. The salermide was dissolved in DMSO and stored at $-20{ }^{\circ} \mathrm{C}$ until use. The same amount of DMSO was used in the final solutions of the test compound as a control. After incubation, the cells were stained with trypan blue and observed under a microscope. For the encystation suppression assays, encystment was induced and the change from cells to cysts was observed under a light microscope. The encystation ratio was calculated by counting the cysts with a hemocytometer under a light microscope after treating the cells with $0.5 \%$ sarkosyl and $0.4 \%$ trypan blue [47].

\section{Statistical analysis}

All statistical analyses were conducted using analysis of variance (ANOVA) and Student's t-tests with GraphPad Prism 8.2 software (GraphPad, San Diego, CA, USA). Significant group differences were evaluated with Tukey's post-hoc multiple comparison tests or Bonferroni correction. $P<0.01(* *)$ was applied to consider significant differences from the control.

\section{Results}

\section{Identification of the $A$. castellanii silent information} regulator 2-like protein

In a previous study, we examined microarray data to identify genes that were differentially expressed in the trophozoite and cyst stages in A. castellanii and found a
cDNA fragment that exhibited sequence homology with SIR2-like proteins [21]. Because a gene for SIR2-like proteins had not previously been found in Acanthamoeba spp., we searched for other SIR2-like proteins by data mining the $A$. castellanii genome database and found four homologues containing the conserved domain of the SIR2 family (pfam02146) but differing in their sequence and length (AcSir2a-d; see Additional file 1: Table S1). Of the four homologues, the deduced amino acid sequence for AcSir2b fully matched that of the cDNA fragment observed in our previous microarray analysis.

To determine whether the four SIR2 homologues were expressed in Acanthamoeba, their expression was assessed using qRT-PCR with cDNA from the trophozoites and cysts. Of the four SIR2 homologues, only AcSir2b was expressed in the trophozoites and cysts (Additional file 1: Figure S1). Using RT-PCR and sequence comparisons of the genome and cDNA sequences, the fulllength sequence for AcSir2b was determined. AcSir2b was designated as A castellanii Sir2-like protein (AcSir2, GenBank: XP_004358245). The AcSir2 gene contains a 1611-bp open reading frame, encoding a 536-amino acidlong protein with a predicted molecular weight of 60.3 $\mathrm{kDa}$. The primary structure of AcSir2 contains a conserved core domain of the SIR2 family of proteins (residues 24-313) including GAGISXXXGIPXXR, PXXXH, TQNID, HG, and two sets of CXXC that may be zinc finger domains (Additional file 1: Figure S2) [48, 49]. The GAWTK motif, which is similar to the conserved GVWTL motif in class-IV sirtuins, was also found in AcSir2. Interestingly, AcSir2 exhibits one structural difference from SIR2 family proteins in other organisms. The YEATS family domain (pfam03366) [50], which recognizes and binds acetylated lysine, is followed by the SIR2 conserved domain of AcSir2 (residues 443-524). The deduced amino acid sequence of AcSir2 exhibited similarities to SIR2 family proteins in other organisms, including Naegleria gruberi (21.9\% sequence identity) [51], the sirtuin $6 \mathrm{~S}$ homolog in Xenopus laevis (22.3\% sequence identity), NAD-dependent deacetylase sirtuin-7 in Chrysochromulina spp. (21.5\% sequence identity), and the SIR2 family transcriptional regulator in Tetrahymena thermophila (21.6\% sequence identity) (Additional file 1: Figure S2). AcSir2 also exhibited a $24 \%$ and $20 \%$ amino acid sequence identity with human SIRT6 and SIRT7, respectively (Additional file 1: Table S2).

Phylogenetic analysis of the conserved deacetylase domains of AcSir2 in comparison with other SIR2 family proteins from various organisms (Additional file 1: Table S3) revealed that AcSir2 clustered in a clade that is closely related to class IV sirtuins including human SIRT6 and SIRT7 (GenBank: NP_057623 and NP_057622, respectively) and fruit fly sirtuin 6 and 


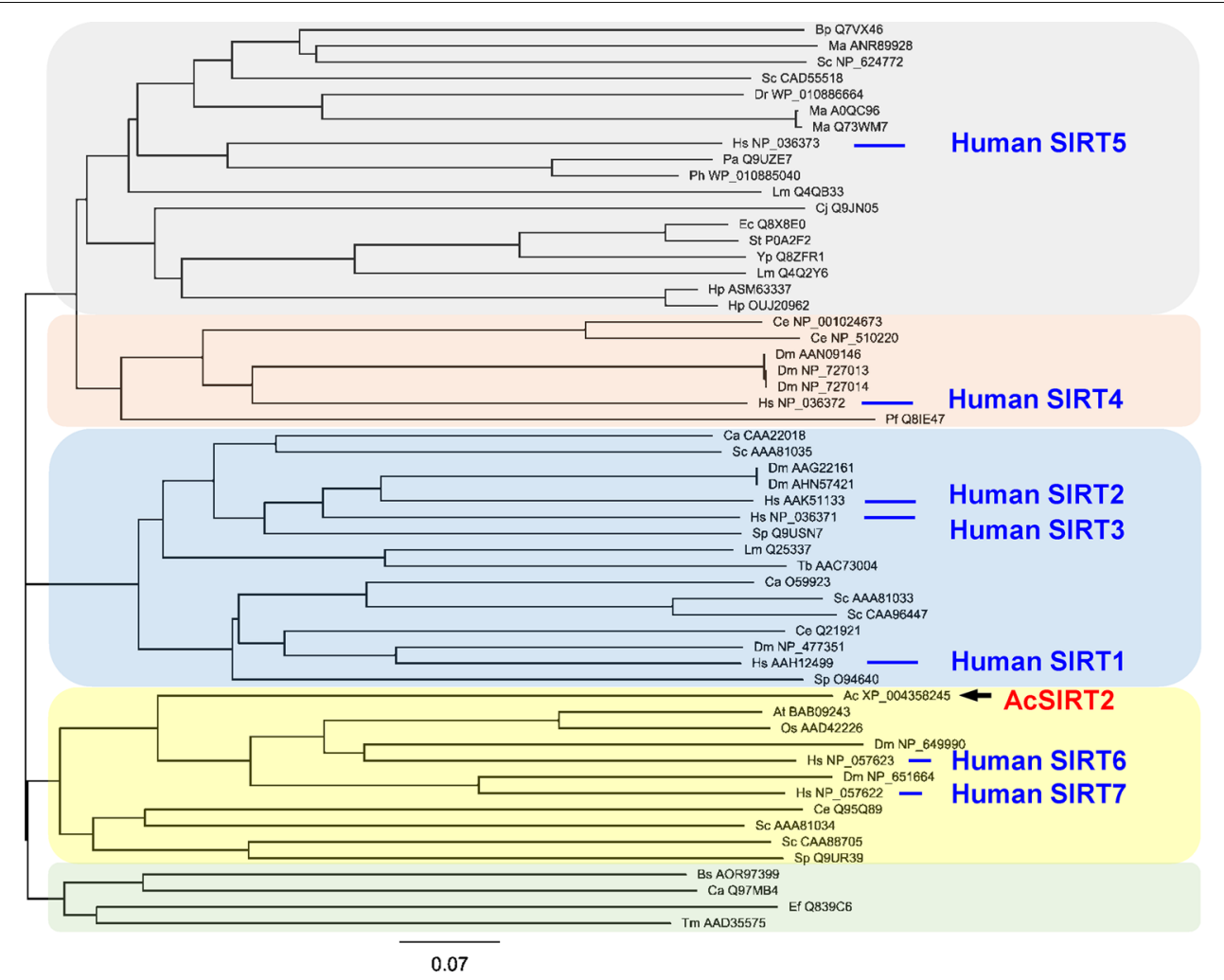

III

Fig. 1 Phylogenetic relationship between AcSir2 and the Sir family in other species. The sequences used in this study are listed in Additional file 1: Table S3

sirtuin 7 (GenBank: NP_649990 and NP_651664, respectively) (Fig. 1).

\section{AcSir2 is a functional SIRT deacetylase in A. castellanii}

To determine whether AcSir2 was functional, we investigated SIRT deacetylase activity in AcSir2-overexpressing Acanthamoeba. AcSir2 was cloned into the GAPDH promoter of the Acanthamoeba expression vector (pGAPDH-EGFP) in order to continuously express AcSir2 using C-terminal EGFP fusion (pGAPDH-AcSir2EGFP). Transfected trophozoites were incubated separately in either PYG medium or encystation medium for $72 \mathrm{~h}$ and examined the transcriptional changes of AcSir2 using qRT-PCR. It was subsequently found that the transcriptional levels of the pGAPDH-AcSir2-EGFP transfectants were approximately 250- and 134-fold higher in the trophozoites and cysts compared with the vector control, respectively $\left(F_{(1,4)}=1186.3, P<0.0001\right.$ and $F_{(1 \text {, }}$ $\left.{ }_{4}\right)=1017.5, P<0.0001$ ) (Additional file 1: Figure S3).

Before determining SIRT deacetylase activity, we examined the intracellular localization of AcSir2 because SIRT2 family proteins are known to differ in their subcellular location. Under a fluorescence microscope after staining with DAPI to label the nucleus, the AcSir2-EGFP fusion protein was mainly found in the nucleus of both the trophozoites and cysts, with lower quantities in the cytoplasm (Fig. 2a). SIRT deacetylase activity was thus measured in the nuclear extracts of AcSir2-overexpressing cells and the vector control. As shown in Fig. 2b, the SIRT deacetylase activity in AcSir2-overexpressing trophozoites was 4 times higher than the vector control $(0.1185 \pm 0.0033$ $\mathrm{OD} / \mathrm{min} / \mathrm{mg}$ vs $0.0265 \pm 0.0012 \mathrm{OD} / \mathrm{min} / \mathrm{mg}$, Tukey's HSD, $P<0.0001)$. This increase was reduced in the absence of the cofactor $\mathrm{NAD}^{+}(0.0678 \pm 0.0032 \mathrm{OD} / \mathrm{min} / \mathrm{mg}$, Tukey's HSD, $P<0.0001)$ or in the presence of the sirtuin inhibitors nicotinamide $(500 \mu \mathrm{M}, 0.0883 \pm 0.0060 \mathrm{OD} /$ $\mathrm{min} / \mathrm{mg}$, Tukey's HSD, $P=0.0011)$ and salermide $(500 \mu \mathrm{M}$, $0.0924 \pm 0.0064 \mathrm{OD} / \mathrm{min} / \mathrm{mg}$, Tukey's HSD, $P=0.0033$ ). Because SIRT deacetylase activity was higher following the overexpression of AcSir2, it can be concluded that AcSir2 is functional SIRT deacetylase.

\section{AcSir2 overexpression leads to trophozoite proliferation}

We observed that AcSir2-overexpressing trophozoites exhibited more rapid growth and larger cell sizes than those in the control. To further examine the effect of AcSir2 overexpression on the growth of trophozoites, non-transfected (i.e. wild-type), EGFP-, and AcSir2-overexpressing cells were seeded at equal densities and the number of cells assessed after 24, 48 and $72 \mathrm{~h}$. As shown 


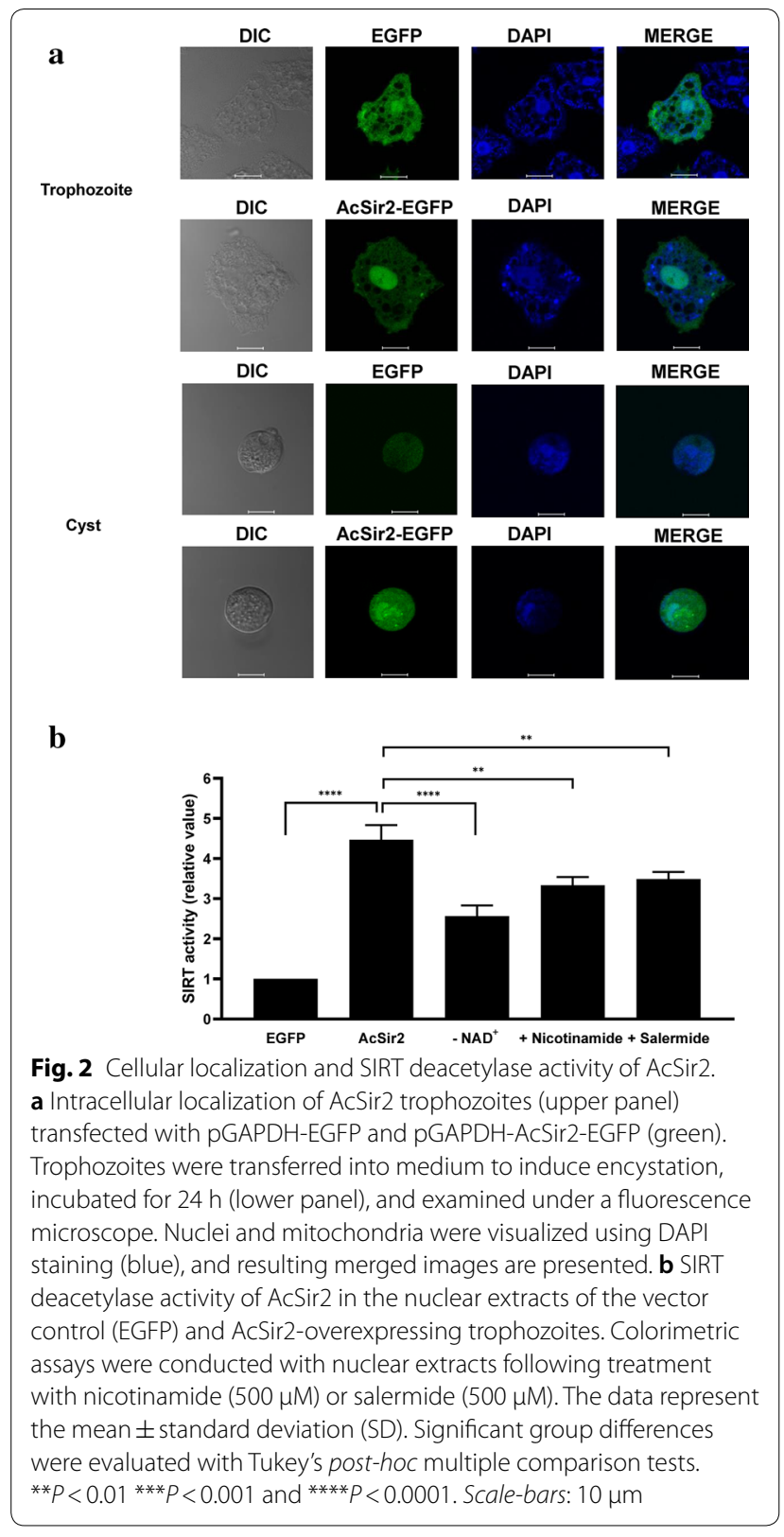

in Fig. 3a, the cell density of the AcSir2-overexpressing trophozoites was higher, showing a 1.63 - and 1.67-fold (Tukey's HSD, $P<0.0001$ ) increase after $72 \mathrm{~h}$ compared to the wild-type and EGFP-overexpressing cells, respectively. In order to confirm that the increase in cell number was due to the overexpression of AcSir2, the cells were treated with various concentrations of the sirtuin inhibitor salermide and the number of cells assessed 24 and $48 \mathrm{~h}$ after treatment. As shown in Fig. 3b, after $24 \mathrm{~h}$, the number of salermide-treated cells increased by $36.7,25.5$ and $0.01 \%$, compared to those of DMSOtreated cells at 50,100 and $200 \mu \mathrm{M}$ salermide, respectively $\left(t_{(4)}=5.97, P=0.004\right)$. After $48 \mathrm{~h}$ of treatment with

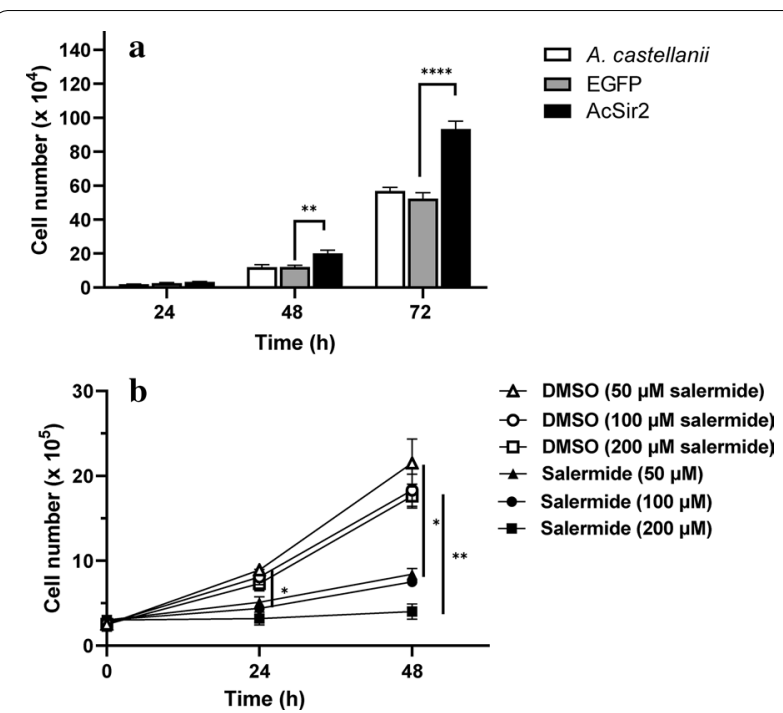

Fig. 3 Effects of AcSir2 overexpression on the growth of A. castellanii trophozoites. a The total cell number of wild-type A. castellanii and cells transfected with pGAPDH-EGFP only and pGAPDH-AcSir2-EGFP plasmids 24, 48 and $72 \mathrm{~h}$ after incubation in PYG culture medium. The data represent the mean cell number \pm standard deviation (SD) $\left.{ }^{* *} P<0.001\right)$. $\mathbf{b}$ Effects of salermide on the proliferation of $A$. castellanii trophozoites. Trophozoites were incubated with various concentrations of salermide (a sirtuin inhibitor) or DMSO (a solvent control). The data represent the mean cell number 0, 24 and 48 $\mathrm{h}$ after incubation for each salermide or DMSO concentration. Significant group differences were evaluated with Tukey's post-hoc multiple comparison tests. ${ }^{* *} P<0.01,{ }^{* * *} P<0.001$ and ${ }^{* * * *} P<0.0001$

$200 \mu \mathrm{M}$ salermide, the difference in cell number became more significant between DMSO- and salermide-treated cells (Tukey's HSD, $P=0.0092$ ), indicating that salermide inhibited the proliferation of $A$. castellanii trophozoites in a dose- and time-dependent manner and that the higher cell density of $A$. castellanii trophozoites was due to AcSir2 overexpression.

Because AcSir2-overexpressing cells exhibited a difference in cell size under a light microscope, we analyzed EGFP and pGAPDH-AcSir2-EGFP transfectants using flow cytometry to quantitatively determine the cell size based on the measurement of mean $\mathrm{FSC}-\mathrm{H}$, a parameter used routinely to measure the size of cells [52]. The overexpression of AcSir2 shifted the mean FSC-H histogram rightward (mean FSC-H = 609.19) compared with the vector control (mean FSC-H = 527.17) (Fig. 4a), indicating that the size of the AcSir2-overexpressing cells was larger by $116 \%$. The EGFP- and AcSir2-overexpressing trophozoites were then stained with propidium iodide (PI) and their DNA content examined using flow cytometry. As shown in Fig. 4b, the FL2 values for the AcSir2-overexpressing cells $(454.74 \pm 30.63)$ exhibited a large rightward shift compared with the control $(159.77 \pm 48.91)$, meaning that the overexpression of AcSir2 in trophozoites 

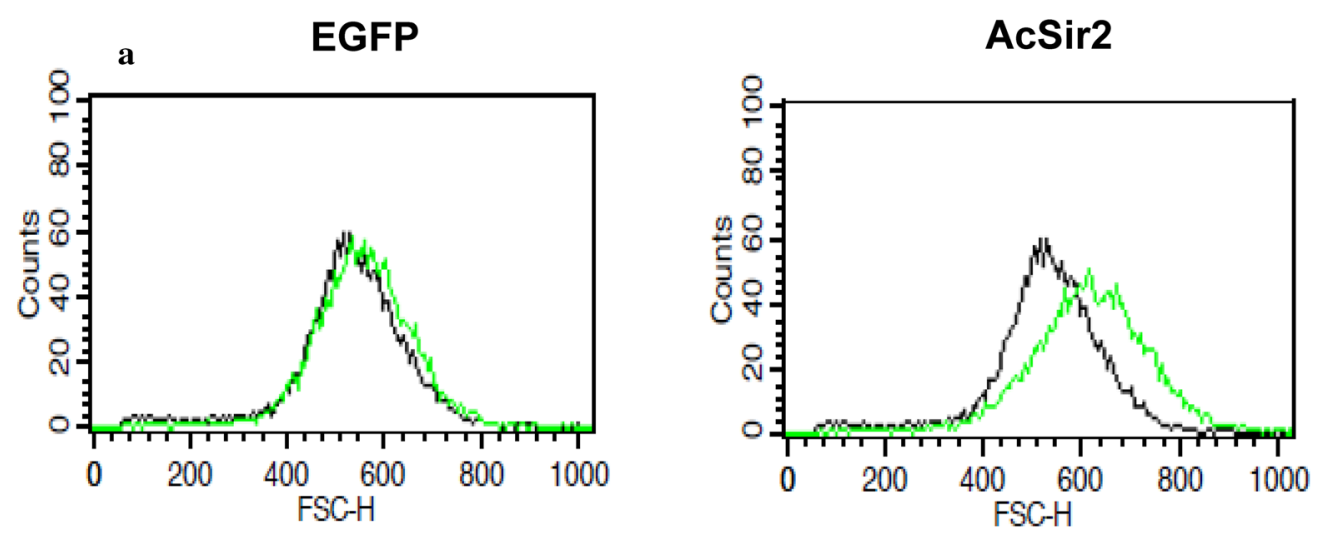

b

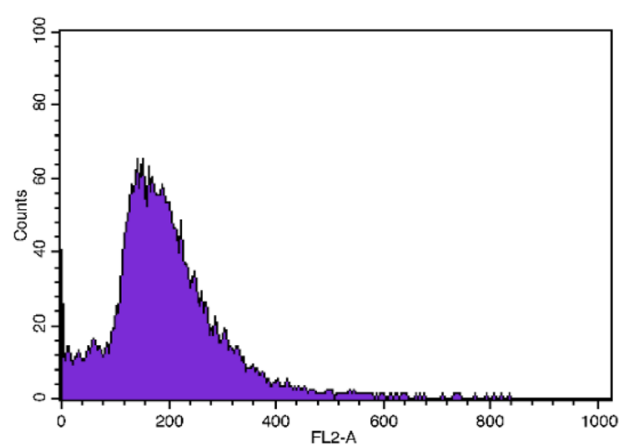

c

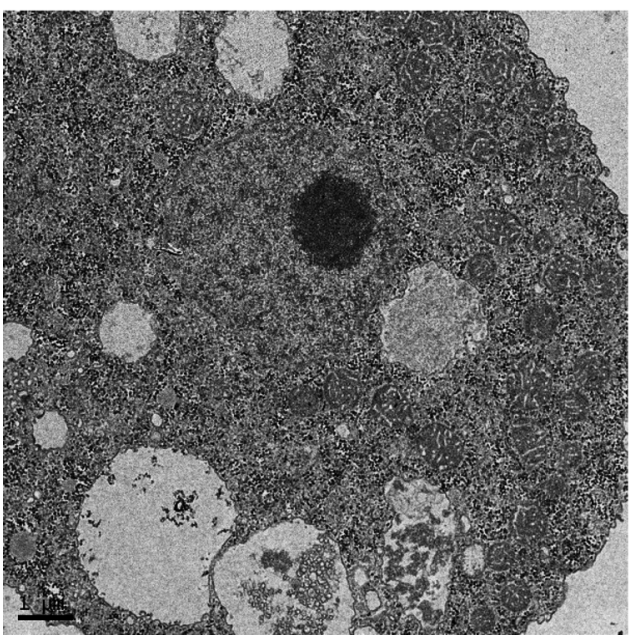

AcSir2

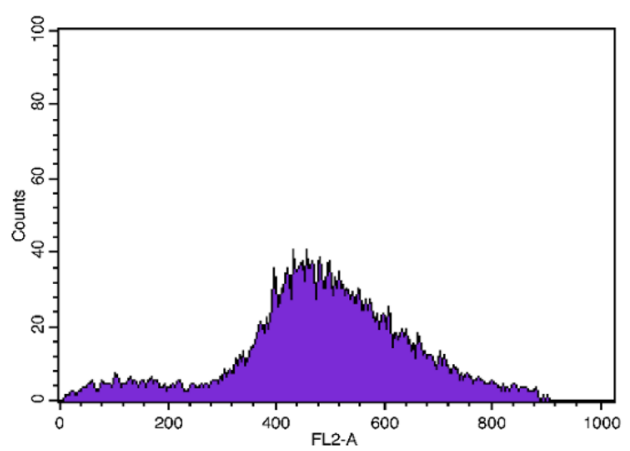

AcSir2

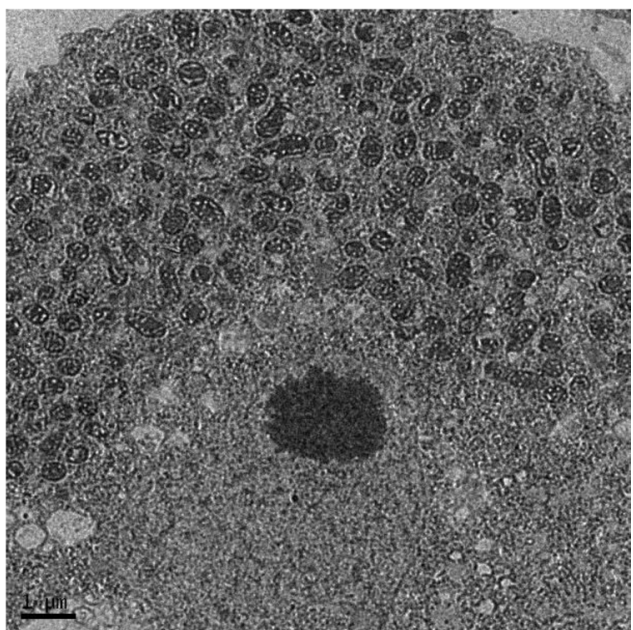

Fig. 4 Effects of AcSir2 overexpression on Acanthamoeba trophozoites. a Wild-type (A. castellanii, black) and trophozoites overexpressing either EGFP (left, green) or AcSir2 (right, green) were analyzed on a flow cytometer for changes in cell size using the mean FSC-H. b The DNA content of the trophozoites overexpressing either EGFP (left) or AcSir2 (right) was determined after PI staining using flow cytometry. The data represent one of three experiments with similar results. c Ultrastructural changes in AcSir2-overexpressing cells. Trophozoites transfected with the vector control (pGAPDH-EGFP) (left) and pGAPDH-AcSir2-EGFP (right) were examined using transmission electron microscopy (TEM). Scale-bars: c, $1 \mu \mathrm{m}$ 
increased the DNA content by $284.6 \%$. Subsequently, the ultrastructural changes in AcSir2-overexpressing cells were examined using TEM. As shown in Fig. 4c, a higher electron density and an increase in the number of mitochondria were observed in AcSir2-overexpressing trophozoites (right) compared with the vector control (left), showing that the overexpression of AcSir2 increased the intracellular content of mitochondria.

\section{AcSir2 is highly expressed during the encystation of $A$. castellanii and its overexpression accelerates the encystation process}

Changes in the expression of AcSir2 in both trophozoites and encysting cells were determined using qRT-PCR. As shown in Fig. 5a, the transcriptional levels of AcSir2 increased approximately 11 -fold at $24 \mathrm{~h}$ post-encystation $(P<0.0001)$; these levels gradually decreased at 48 and $72 \mathrm{~h}$ post-encystation, but remained higher than those in trophozoites. Under a light microscope, we observed that a larger number of cysts formed in the AcSir2overexpressing cells than in the control cells after the induction of encystation (Fig. 5b). To ascertain whether greater AcSir2 expression affects the encystation of $A$. castellanii, vector control and AcSir2-overexpressing trophozoites were transferred to encystation medium to induce encystation and incubated for 24,48 and $72 \mathrm{~h}$, and mature cysts were counted under a microscope after treatment with sarkosyl. As shown in Fig. 5c, both the vector control and AcSir2-overexpressing cells encysted gradually over time, though the encystation rate was significantly higher in the latter group. In particular, at 48 h post-encystation, $45.3 \%$ of the vector control cells had transformed into mature cysts, compared to $82.5 \%$ of the AcSir2-overexpressing cells (post-hoc pairwise tests with Bonferroni correction; $P=0.0002$ ).

We then looked to verify whether the more rapid encystation was specifically due to the overexpression of AcSir2. The encysting trophozoites were treated with various concentrations of salermide and the encystation rate determined. As shown in Fig. 5d, $49.8 \%$ of cells treated with $100 \mu \mathrm{M}$ of salermide transformed into cysts at $72 \mathrm{~h}$ post-encystation, which was significantly lower than DMSO-treated control cells $\left(t_{(4)}=5.119, P=0.0069\right)$. This indicates that sirtuin inhibitors suppress the encystation of $A$. castellanii and that encystation is accelerated by the overexpression of AcSir2.

\section{Ultrastructural changes occur following treatment with a sirtuin inhibitor in encysting $A$. castellanii} We then examined the morphological changes in encysting cells treated with a sirtuin inhibitor. Differences between DMSO- and salermide-treated $(100 \mu \mathrm{M})$ cells were examined using TEM. The DMSO-treated cells transformed into mature double-walled cysts with a laminar fibrous ectocyst wall (Fig. 6a, left, filled arrowheads) and an endocyst wall composed of fine fibrils in a granular matrix (Fig. 6a, left, open arrowheads), which is a morphology typical of Acanthamoeba cysts [5]. Vesicular structures were also detected, and the partial degradation of organelles and intracellular compartments within these structures was observed at $24 \mathrm{~h}$ post-encystation (Fig. 6a, left). In comparison, when A. castellanii cells were transferred into encystation medium containing $100 \mu \mathrm{M}$ salermide for $24 \mathrm{~h}$, the ectocyst wall was observed to be deeply wrinkled and thicker than that of the DMSO-treated cells, which exhibited thorn-like spines protruding from the cytoplasm (Fig. 6a, right). The endocyst wall and intercyst space separating the ectocyst and endocyst walls (Fig. 6a, left, double-headed arrow), which are typically observed in encysting Acanthamoeba, were noticeably absent in the salermide-treated encysting cells (Fig. 6a, right), suggesting that salermide significantly impairs the formation of the cyst wall during the encystation process.

\section{The transcriptional regulation of $A$. castellanii encystation-mediating proteins}

To investigate the cause of the more rapid encystation in AcSir2-overexpressing cells and the impaired formation of the cyst wall in salermide-treated $A$. castellanii cells, we first examined the transcriptional levels of cellulose synthase, which is known to be upregulated in encysting cells [17]. The transcriptional levels of cellulose synthase in AcSir2-overexpressing cells were 3.1- and 7.2-fold higher at 24 and $48 \mathrm{~h}$ after the induction of encystation, respectively, compared with vector control cells (Tukey's HSD, $P<0.0001$ ) (Fig. 6b), while the transcription of cellulose synthase was completely abolished in salermidetreated cells $\left(t_{(4)}=5.954, P=0.004\right) \quad$ (Fig. 6c). These results show that sirtuin regulates the transcriptional levels of cellulose synthase during encystation.

\section{Discussion}

In mammals, there are seven sirtuin isoforms (Sirt1-7), and sirtuins have been classified into five major classes (I, II, III, IV and U) that differ in their intracellular distribution $[27,49,53,54]$. Phylogenetic analysis comparing the conserved catalytic domain of AcSir2 with the sirtuins reported from various organisms revealed that AcSir2 is a class IV sirtuin, as are SIRT6 and SIRT7, which are found in humans, fruit flies and plants (Fig. 1). Consistent with initial reports on the nuclear localization of human SIRT6 and SIRT7 [25], EGFP-tagged AcSir2 was predominantly localized in the nucleus. In protozoan parasites, 
a

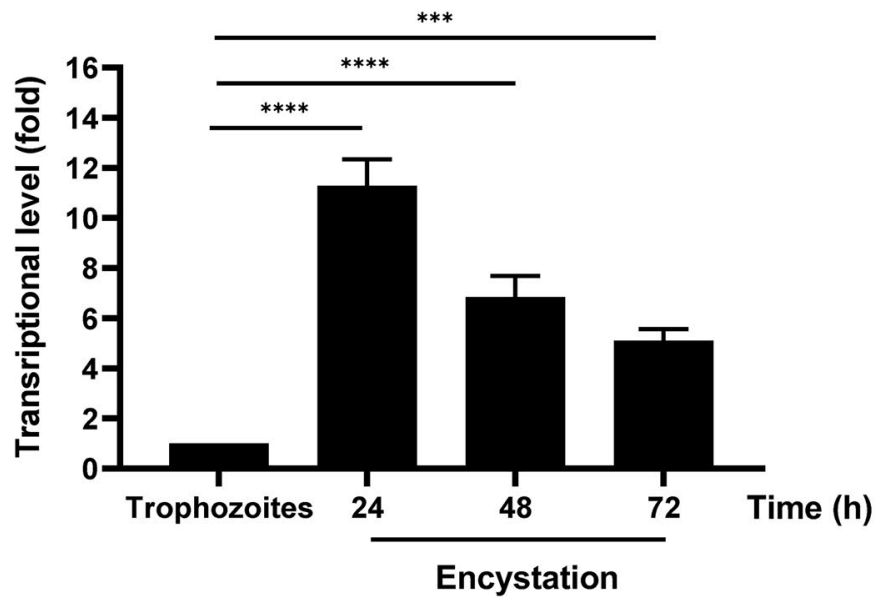

b

\section{Encysting cells $(48 \mathrm{~h})$}
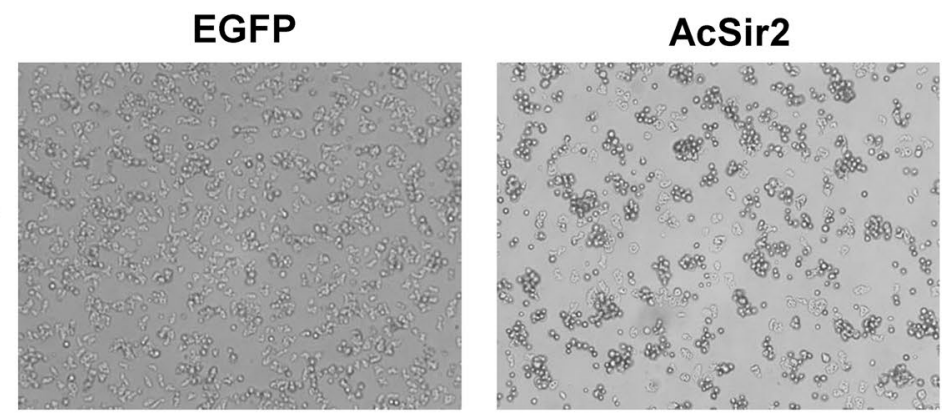

c

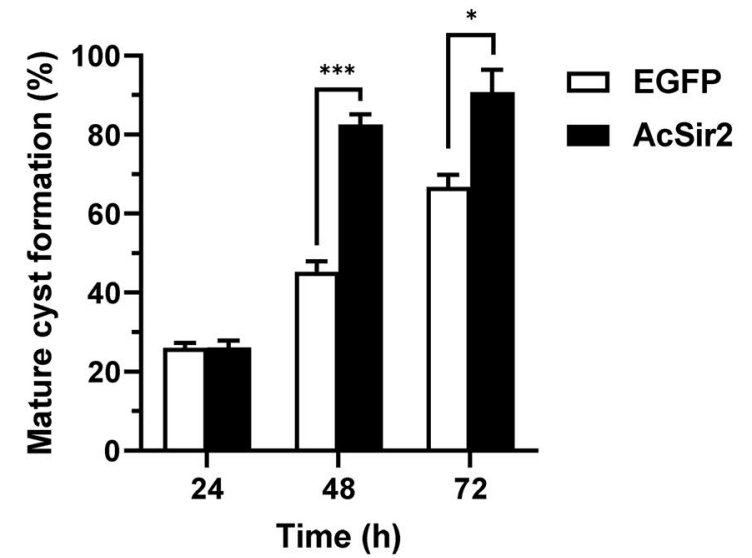

d

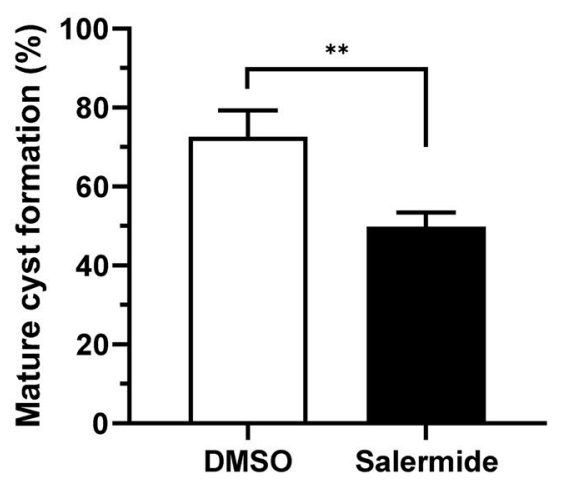

Fig. 5 Transcriptional changes in AcSir2 during encystation and the effects of AcSir2 overexpression on the encystation of A. castellanii. a Changes in the expression of AcSir2 during the encystation of A. castellanii. Trophozoites and encysting cells 24,48 and $72 \mathrm{~h}$ after the induction of encystation were examined in terms of AcSir2 transcriptional changes using qRT-PCR. Acanthamoeba actin was used as an internal control. b, c Effects of AcSir2 overexpression on the encystation of A. castellanii. Vector control (left) and AcSir2-overexpressing trophozoites (right) were transferred into encystation medium, incubated for $48 \mathrm{~h}$, and examined directly under a microscope (b). Encysting cells overexpressing EGFP and AcSir2 at 24, 48 and $72 \mathrm{~h}$ after treatment with $0.5 \%$ sarkosyl for $30 \mathrm{~min}$ and staining with $0.4 \%$ trypan blue, with the number of mature cysts counted under a microscope (c). $\mathbf{d}$ Effects of salermide on the encystation of A. castellanii trophozoites. A. castellanii trophozoites were transferred into encystation medium containing $100 \mu \mathrm{M}$ of salermide and incubated for $72 \mathrm{~h}$. The mature cysts were counted under a microscope, followed by treatment with sarkosyl. DMSO was used as a solvent control. The percentage of mature cysts is calculated as the mean number of cells remaining after sarkosyl treatment compared with the total number of cells. The data represent the mean \pm standard deviation (SD) of three separate experiments. ${ }^{* *} P<0.01,{ }^{* * *} P<0.001$ and ${ }^{* * *} P<0.0001$ 


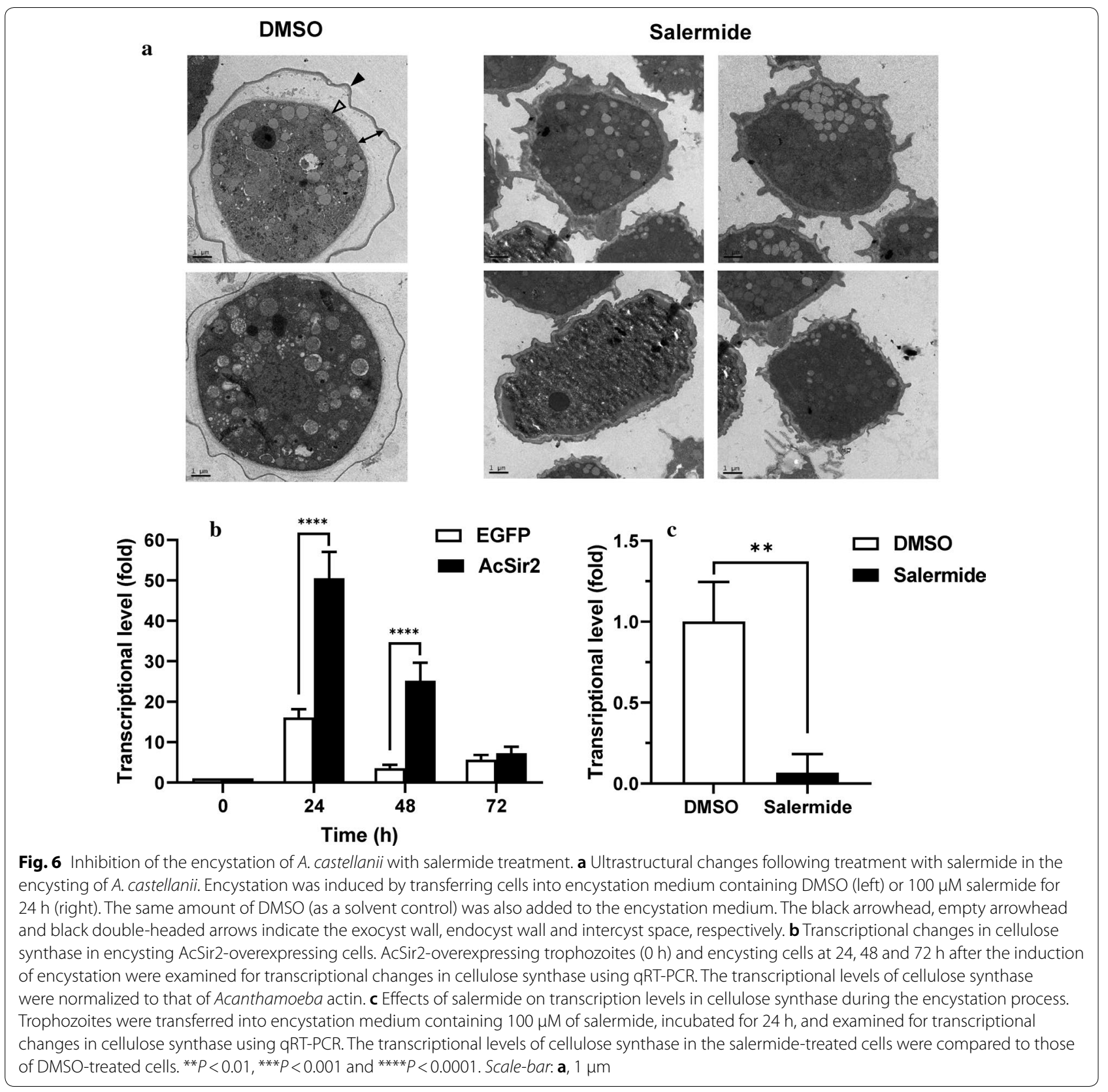

class-IV sirtuins have previously only been found in the phylum Apicomplexa (Plasmodium, Toxoplasma, Neospora, Theileria, Babesia and Eimeria), while class I, II, III, and U sirtuins have been reported in the phylum Sarcomastigophora, such as Trypanosoma, Leishmania, Giardia and Trichomonas (see [33] for a review). Thus, AcSir2 is the first class IV sirtuin to be reported in Sarcomastigophora. Interestingly, AcSir2 has a YEATS domain, which is named for the five proteins that contain this domain (Yaf9, ENL, Af9, Taf14 and Sas5) and which recognizes and binds acetylated lysine. The YEATS domain functions as a docking site for reader proteins and is evolutionarily conserved in organisms ranging from yeast to humans. This domain is observed in a variety of nuclear complexes with molecular functions ranging from histone modification to transcription regulation $[55,56]$. However, no YEATS domain has been observed in SIR2-like proteins in other organisms. The AcSir2 protein contains the YEATS domain followed by a Sir2 catalytic domain that functions as an $\mathrm{NAD}^{+}$-dependent deacetylase. This structural characteristic may contribute to more effective SIRT deacetylase activity. This structure is also likely to be a primitive form of $\mathrm{NAD}^{+}$-dependent deacetylase, supposing the evolutionary divergence 
into sirtuin family proteins with the Sirt2 domain and transcription-regulating proteins with only the YEATS domain. Further research using YEATS domain deletion mutants of AcSir2 is required to examine SIRT deacetylase activity and compare the effects on encystation with wild-type AcSir2.

Mammalian SIRT1-3 have strong deacetylase activity, while SIRT4-7 are known to have weak or nondetectable deacetylase activity [57]. In the present study, nuclear extracts of wild-type $A$. castellanii trophozoites exhibited non-detectable deacetylase activity (Fig. 2b). Thus, we examined the deacetylase activity in AcSir2overexpressing cells. Incubation of the nuclear extracts of AcSir2-overexpressing cells with an acetylated substrate in the presence of NAD+ cofactor exhibited deacetylase activity (Fig. 2b). As shown in Fig. 2b, the greater SIRT deacetylase activity due to the overexpression of AcSir2 was weakened in the presence of nicotinamide, one of the products of the deacetylation reaction [58], and salermide, a sirtuin inhibitor, verifying that AcSir2 has SIRT deacetylase activity.

In this study, A. castellanii AcSir2-overexpressing trophozoites had greater cell density compared with the vector control (Fig. 3a). In salermide-treated AcSir2 overexpressing cells, proliferation of trophozoites was not significantly inhibited due to the residual SIRT deacetylase activity (Fig. 2b and Additional file 1: Figure S4). Salermide suppressed the growth of wild type A. castellanii trophozoites in a dose- and time-dependent manner (Fig. 3b). These results indicate that the higher cell density was a result of the overexpression of AcSir2. In yeast, worms and flies, Sir2 homologs positively regulate longevity [59-63], while Ford et al. [64] suggested that SIRT7 is a nuclear protein that positively regulates RNA polymerase I and is required for cell viability in mammals. In actively proliferating trophozoites, the higher cell density could be attributed to either more rapid cell division or increased longevity; which of these mechanisms is dominant has not yet been clarified for AcSir2overexpressing cells. Also, the used plasmid vectors were reported to be maintained episomally [45]; we open the possibility that cell death could be delayed due to deleterious mutations or homologous recombination by transfected plasmids.

The AcSir2-overexpressing cells were also observed to be larger in size, with a rightward shift observed in the FSC-H histograms when compared with the control (Fig. 4a). To examine the reason for this increase in cell size, DNA staining using PI revealed higher DNA content in AcSir2-overexpressing cells. In the TEM analysis, ultrastructural changes in AcSir2-overexpressing cells indicates that this increase in DNA content was due to an increase in the number of mitochondria (Fig. 4c).
Thus, it is likely that the increase in cell size of AcSir2overexpressing cells was caused by the increase in mitochondrial DNA. In mammals, SIRT1, which is primarily nuclear, has been implicated in mitochondrial biogenesis via the deacetylation of target proteins such as hypoxiainducible factor $1 \alpha(\mathrm{HIF}-1 \alpha)$ [65] and peroxisome proliferator-activated receptor gamma coactivator-1 (PGC-1) [66]. When searching the Acanthamoeba genome, we only found a homologue of HIF- $1 \alpha$, thus further studies on the deacetylation of target proteins by AcSir2 are required to determine its importance in mitochondrial biogenesis.

Sir2-like proteins, which are $\mathrm{NAD}^{+}$-dependent deacetylases, sense intracellular levels of $\mathrm{NAD}^{+}$, which are affected by caloric uptake $[67,68]$, and communicate the nutrient status of the external environment to the different intracellular compartments within the cell. The encystation of Acanthamoeba occurs to ensure its survival and transmission in a variety of adverse environmental conditions, including the depletion of nutrients. In the present study, although it remains unknown whether AcSir2 acts as a nutrient and metabolic sensor in A. castellanii, the transcription of AcSir2 was upregulated during encystation (Fig. 5a). The overexpression of AcSir2 following transfection with an AcSir2-cloned $A$. castellanii expression vector accelerated the encystation process, converting cells into mature cysts more efficiently (Fig. 5b, c), while the encystation of A. castellanii was suppressed by salermide treatment (Fig. 5d). Previous research has shown that, in S. cerevisiae, SIR2 levels increase during calorie restriction [69, 70], and sirtuin overexpression is known to extend the lifespan by silencing HML and HMR loci and inhibiting the formation of extrachromosomal rDNA circles [71, 72]. Of the T. cruzi sirtuins, TcSir2rp3 not only increases parasite invasion and multiplication, but the overexpression of TcSir2rp3 enhances parasite growth and increases the transformation of epimastigotes to metacyclic trypomastigotes due to starvation [36]. In addition, as in A. castellanii, salermide reduced the growth and differentiation of $T$. cruzi. Despite this mass of evidence for the importance of sirtuins in the encystation process, further research is needed to determine what changes occur at the intracellular level during encystation in AcSir2-overexpressed cells. In addition, it has been reported that the Acanthamoeba strain used in this study loses its potential for encystment by long-term culture under axenic condition [73]. Therefore, the effects of salermide treatments on encystation should be evaluated in fresh isolates or other strains of Acanthamoeba.

Acanthamoeba cysts have an ectocyst wall (the outer cyst wall) consisting of acid-insoluble protein-containing materials and an endocyst wall (the inner cyst wall), 
which is partially composed of cellulose $[3,7,8]$. Recent studies have shown that cellulose fibrils exist in both cyst walls, and cellulose synthesis has been suggested as an important target for the treatment of Acanthamoeba infections [74]. Previous research on cellulose synthesis inhibitors and the knockdown of Acanthamoeba cellulose synthase has found that cellulose is essential to the formation of cyst walls during encystation $[17,75]$. It is known that the transcriptional levels of cellulose synthase are upregulated during the encystation process [76]. In the present study, the transcriptional increase in cellulose synthase in AcSir2-overexpressing cells was more significant than in the control (Fig. 6b). However, the transcription of cellulose synthase was completely abolished in salermide-treated cells (Fig. 6c). In TEM analysis, we found that the structure of the ectocyst wall, intercyst space, and endocyst wall was subsequently impaired by salermide treatment. In particular, the endocyst wall, which consists of polysaccharides such as cellulose [4, 77], was absent in salermide-treated encysting cells (Fig. 6a). Thus, it is likely that that the absence of endocysts in the salermide-treated cells was due to the absence of cellulose arising from the lack of cellulose synthase.

\section{Conclusions}

We identified the sirtuin-like protein AcSir2 in A. castellanii, which, to the best of our knowledge, is the first class-IV sirtuin reported in the phylum Sarcomastigophora. AcSir2 has functional SIRT deacetylase activity and is localized primarily in the nucleus. AcSir2 overexpression leads to increased cell growth in A. castellanii trophozoites and accelerated encystation, and these changes were reverted by salermide treatment. The regulation of AcSir2 expression is found to be important for the growth and encystation of Acanthamoeba, thus highlighting its potential worth as a therapeutic target.

\section{Supplementary information}

Supplementary information accompanies this paper at https://doi. org/10.1186/s13071-020-04237-5.

\footnotetext{
Additional file 1: Table S1. The predicted amino acid sequences of the genes with the conserved domain of SIR2 family proteins following the screening of the Acanthamoeba genome database. Figure S1. Transcriptional profiles were obtained with qRT-PCR for four SIR2 homologues using CDNAs from A. castellanii trophozoites ( $T$ ) and encysting cells $(C)$ at 24,48 , and $72 \mathrm{~h}$ after induction of encystation. Figure S2. Comparison of AcSir2 (XP_004358245) with sirtuin-like proteins from other organisms. Table S2. Distance matrix of the identity scores (\%) resulting from the alignment of AcSir2 with human sirtuin proteins. Table S3. GenBank accession numbers and description of Sir2 family proteins in terms of multiple alignment and phylogeny. Figure S3. Overexpression of ACSir2 in A. castellanii trophozoites and cysts. Figure S4. Effects of salermide on proliferation of $A$. castellanii trophozoites (upper), and EGFP- (middle) and AcSir2-EGFP (lower) overexpressing trophozoites.
}

\section{Abbreviations}

AcSir2: Acanthamoeba castellanii sirtuin 2; NAD: nicotinamide nucleotide dinucleotide; EGFP: enhanced green fluorescent protein; RT-PCR: reverse transcription-polymerase chain reaction; qRT-PCR: quantitative real-time PCR; TEM: transmission electron microscopy; PI: propidium iodide; FSC-H: forward scatter height; HIF-1a: hypoxia-inducible factor 1a; PGC-1: peroxisome proliferator-activated receptor Gamma Coactivator-1.

\section{Acknowledgements \\ Not applicable.}

\section{Authors' contributions}

SYJ contributed to the design, analysis, completion, interpretation and reporting of the study. MS participated in one or more of the experiments. JMA, YKG, DIC, EKM and HHK analyzed the data and interpreted the results. All authors read and approved the final manuscript.

\section{Funding}

This work was supported by a National Research Foundation of Korea (NRF) grant funded by the Korean government (MEST)(NRF-2019R1A2C1004177).

The funders had no role in the study design, data collection, analysis, decision to publish, or preparation of the manuscript.

\section{Availability of data and materials}

The data supporting the conclusions of this article are included within the article and its additional file. The plasmids are available on request.

\section{Ethics approval and consent to participate}

Not applicable.

\section{Consent for publication \\ Not applicable.}

\section{Competing interests}

The authors declare that they have no competing interests.

\section{Author details}

${ }^{1}$ Department of Parasitology and Tropical Medicine, School of Medicine, Kyungpook National University, Daegu, Republic of Korea. ${ }^{2}$ Department of Microbiology, School of Medicine, Kyungpook National University, Daegu, Republic of Korea. ${ }^{3}$ Department of Medical Zoology, Kyung Hee University School of Medicine, Seoul, Republic of Korea. ${ }^{4}$ Department of Parasitology, Dong-A University College of Medicine, Busan, Republic of Korea.

Received: 26 April 2020 Accepted: 15 July 2020

Published online: 22 July 2020

\section{References}

1. Khan NA. Acanthamoeba: biology and pathogenesis. 2nd ed. Norfolk: Caister Academic Press; 2015.

2. Walochnik J, Duchêne M. Molecular parasitology: protozoan parasites and their molecules. Wien: Springer; 2016.

3. Lloyd D, Turner NA, Khunkitti W, Hann AC, Furr JR, Russell AD. Encystation in Acanthamoeba castellanii: development of biocide resistance. J Eukaryot Microbiol. 2001;48:11-6.

4. Tomlinson $\mathrm{G}$, Jones EA. Isolation of cellulose from the cyst wall of a soil amoeba. Biochim Biophys Acta. 1962;63:194-200.

5. Weisman RA. Differentiation in Acanthamoeba castellanii. Annu Rev Microbiol. 1976;30:189-219.

6. Marciano-Cabral F, Cabral G. Acanthamoeba spp. as agents of disease in humans. Clin Microbiol Rev. 2003;16:273-307.

7. Neff RJ, Neff RH. The biochemistry of amoebic encystment. Symp Soc Exp Biol. 1969;23:51-81.

8. Dudley R, Jarroll EL, Khan NA. Carbohydrate analysis of Acanthamoeba castellanii. Exp Parasitol. 2009;122:338-43.

9. Hirukawa $Y$, Nakato H, Izumi S, Tsuruhara T, Tomino S. Structure and expression of a cyst specific protein of Acanthamoeba castellanii. Biochim Biophys Acta. 1998;1398:47-56. 
10. Moon EK, Chung DI, Hong YC, Kong HH. Characterization of a serine proteinase mediating encystation of Acanthamoeba. Eukaryot Cell. 2008:7:1513-7.

11. Lee $Y R, N a$ BK, Moon EK, Song SM, Joo SY, Kong HH, et al. Essential role for an M17 leucine aminopeptidase in encystation of Acanthamoeba castellanii. PLoS One. 2015;10:e0129884

12. Leitsch D, Kohsler M, Marchetti-Deschmann M, Deutsch A, Allmaier G, Duchene $\mathrm{M}$, et al. Major role for cysteine proteases during the early phase of Acanthamoeba castellanii encystment. Eukaryot Cell. 2010;9:611-8.

13. Moon EK, Hong Y, Chung DI, Kong HH. Cysteine protease involving in autophagosomal degradation of mitochondria during encystation of Acanthamoeba. Mol Biochem Parasitol. 2012;185:121-6.

14. Moon EK, Chung DI, Hong YC, Kong HH. Autophagy protein 8 mediating autophagosome in encysting Acanthamoeba. Mol Biochem Parasitol. 2009;168:43-8.

15. Song SM, Han BI, Moon EK, Lee YR, Yu HS, Jha BK, et al. Autophagy protein 16-mediated autophagy is required for the encystation of Acanthamoeba castellanii. Mol Biochem Parasitol. 2012;183:158-65.

16. Moon EK, Chung DI, Hong Y, Kong HH. Atg3-mediated lipidation of Atg8 is involved in encystation of Acanthamoeba. Korean J Parasitol. 2011:49:103-8.

17. Moon EK, Hong Y, Chung DI, Goo YK, Kong HH. Down-regulation of cellulose synthase inhibits the formation of endocysts in Acanthamoeba. Korean J Parasitol. 2014;52:131-5.

18. Potter JL, Weisman RA. Correlation of cellulose synthesis in vivo and in vitro during the encystment of Acanthamoeba. Dev Biol. 1972;28:472-7.

19. Dudley R, Alsam S, Khan NA. Cellulose biosynthesis pathway is a potential target in the improved treatment of Acanthamoeba keratitis. Appl Microbiol Biotechnol. 2007;75:133-40.

20. Lorenzo-Morales J, Kliescikova J, Martinez-Carretero E, De Pablos LM, Profotova B, Nohynkova E, et al. Glycogen phosphorylase in Acanthamoeba spp.: determining the role of the enzyme during the encystment process using RNA interference. Eukaryot Cell. 2008;7:509-17.

21. Moon EK, Xuan YH, Chung DI, Hong Y, Kong HH. Microarray analysis of differentially expressed genes between cysts and trophozoites of Acanthamoeba castellanii. Korean J Parasitol. 2011;49:341-7.

22. Imai S, Armstrong CM, Kaeberlein M, Guarente L. Transcriptional silencing and longevity protein Sir2 is an NAD-dependent histone deacetylase. Nature. 2000;403:795-800

23. Landry J, Sutton A, Tafrov ST, Heller RC, Stebbins J, Pillus L, et al. The silencing protein SIR2 and its homologs are NAD-dependent protein deacetylases. Proc Natl Acad Sci USA. 2000:97:5807-11.

24. Chang HC, Guarente L. SIRT1 and other sirtuins in metabolism. Trends Endocrinol Metab. 2014;25:138-45.

25. Michishita E, Park JY, Burneskis JM, Barrett JC, Horikawa I. Evolutionarily conserved and nonconserved cellular localizations and functions of human SIRT proteins. Mol Biol Cell. 2005;16:4623-35.

26. Kim SC, Sprung R, Chen Y, Xu Y, Ball H, Pei J, et al. Substrate and functional diversity of lysine acetylation revealed by a proteomics survey. Mol Cell. 2006;23:607-18

27. Haigis MC, Sinclair DA. Mammalian sirtuins: biological insights and disease relevance. Annu Rev Pathol. 2010;5:253-95.

28. Sack MN, Finkel T. Mitochondrial metabolism, sirtuins, and aging. Cold Spring Harb Perspect Biol. 2012;4:a013102.

29. Michan S, Sinclair D. Sirtuins in mammals: insights into their biological function. Biochem J. 2007;404:1-13.

30. Houtkooper RH, Pirinen E, Auwerx J. Sirtuins as regulators of metabolism and healthspan. Nat Rev Mol Cell Biol. 2012;13:225-38.

31. Haigis MC, Guarente LP. Mammalian sirtuins - emerging roles in physiology, aging, and calorie restriction. Genes Dev. 2006;20:2913-21.

32. Libert S, Guarente L. Metabolic and neuropsychiatric effects of calorie restriction and sirtuins. Annu Rev Physiol. 2013;75:669-84.

33. Religa AA, Waters AP. Sirtuins of parasitic protozoa: in search of function(s). Mol Biochem Parasitol. 2012;185:71-88.

34. Hailu GS, Robaa D, Forgione M, Sippl W, Rotili D, Mai A. Lysine deacetylase inhibitors in parasites: past, present, and future perspectives. J Med Chem. 2017;60:4780-804.

35. Vergnes B, Vanhille L, Ouaissi A, Sereno D. Stage-specific antileishmanial activity of an inhibitor of SIR2 histone deacetylase. Acta Trop. 2005:94:107-15.
36. Moretti NS, da Silva Augusto L, Clemente TM, Antunes RP, Yoshida N, Torrecilhas AC, et al. Characterization of Trypanosoma cruzi sirtuins as possible drug targets for Chagas disease. Antimicrob Agents Chemother. 2015;59:4669-79.

37. Pierce RJ, Dubois-Abdesselem F, Lancelot J, Andrade L, Oliveira G. Targeting schistosome histone modifying enzymes for drug development. Curr Pharm Des. 2012;18:3567-78.

38. Lancelot J, Caby S, Dubois-Abdesselem F, Vanderstraete M, Trolet J, Oliveira G, et al. Schistosoma mansoni sirtuins: characterization and potential as chemotherapeutic targets. PLoS Negl Trop Dis. 2013;7:e2428.

39. Visvesvara GS, Balamuth W. Comparative studies on related free-living and pathogenic amebae with special reference to Acanthamoeba. J Protozool. 1975;22:245-56.

40. Bowers B, Korn ED. The fine structure of Acanthamoeba castellanii (Neff strain). II. Encystment. J Cell Biol. 1969;41:786-805.

41. Mazur T, Zozwiak M. Value of the color test in assessing the viability of cysts of Acanthamoeba sp. Wiad Parazytol. 1989;35:11-7.

42. Coulon C, Collignon A, McDonnell G, Thomas V. Resistance of Acanthamoeba cysts to disinfection treatments used in health care settings. J Clin Microbiol. 2010;48:2689-97.

43. Aurrecoechea C, Barreto A, Brestelli J, Brunk BP, Caler EV, Fischer S, et al. AmoebaDB and MicrosporidiaDB: functional genomic resources for Amoebozoa and Microsporidia species. Nucleic Acids Res. 2011:39:D612-9.

44. Nellen W, Gallwitz D. Actin genes and actin messenger RNA in Acanthamoeba castellanii. Nucleotide sequence of the split actin gene I. J Mol Biol. 1982;159:1-18.

45. Bateman E. Expression plasmids and production of EGFP in stably transfected Acanthamoeba. Protein Expr Purif. 2010;70:95-100.

46. Yitzhaki S, Barnea A, Keysary A, Zahavy E. New approach for serological testing for leptospirosis by using detection of leptospira agglutination by flow cytometry light scatter analysis. J Clin Microbiol. 2004:42:1680-5.

47. Picazarri K, Nakada-Tsukui K, Nozaki T. Autophagy during proliferation and encystation in the protozoan parasite Entamoeba invadens. Infect Immun. 2008;76:278-88.

48. Brachmann CB, Sherman JM, Devine SE, Cameron EE, Pillus L, Boeke JD. The SIR2 gene family, conserved from bacteria to humans, functions in silencing, cell cycle progression, and chromosome stability. Genes Dev. 1995;9:2888-902

49. Frye RA. Phylogenetic classification of prokaryotic and eukaryotic Sir2like proteins. Biochem Biophys Res Commun. 2000;273:793-8.

50. Li Y, Wen H, Xi Y, Tanaka K, Wang H, Peng D, et al. AF9 YEATS domain links histone acetylation to DOT1L-mediated H3K79 methylation. Cell. 2014;159:558-71.

51. Fritz-Laylin LK, Prochnik SE, Ginger ML, Dacks JB, Carpenter ML, Field $M C$, et al. The genome of Naegleria gruberi illuminates early eukaryotic versatility. Cell. 2010;140:631-42.

52. Vermes I, Haanen C, Reutelingsperger C. Flow cytometry of apoptotic cell death. J Immunol Methods. 2000;243:167-90.

53. Horio Y, Hayashi T, Kuno A, Kunimoto R. Cellular and molecular effects of sirtuins in health and disease. Clin Sci (Lond). 2011:121:191-203.

54. Cen Y, Youn DY, Sauve AA. Advances in characterization of human sirtuin isoforms: chemistries, targets and therapeutic applications. Curr Med Chem. 2011;18:1919-35.

55. Schulze JM, Wang AY, Kobor MS. YEATS domain proteins: a diverse family with many links to chromatin modification and transcription. Biochem Cell Biol. 2009;87:65-75.

56. Schulze JM, Wang AY, Kobor MS. Reading chromatin: insights from yeast into YEATS domain structure and function. Epigenetics. 2010;5:573-7.

57. North BJ, Marshall BL, Borra MT, Denu JM, Verdin E. The human Sir2 ortholog, SIRT2, is an NAD+-dependent tubulin deacetylase. Mol Cell. 2003;11:437-44.

58. Bitterman KJ, Anderson RM, Cohen HY, Latorre-Esteves M, Sinclair DA. Inhibition of silencing and accelerated aging by nicotinamide, a putative negative regulator of yeast sir2 and human SIRT1. J Biol Chem. 2002;277:45099-107. 
59. Friedman DB, Johnson TE. Three mutants that extend both mean and maximum life span of the nematode, Caenorhabditis elegans, define the age-1 gene. J Gerontol. 1988;43:B102-9.

60. Friedman DB, Johnson TE. A mutation in the age-1 gene in Caenorhabditis elegans lengthens life and reduces hermaphrodite fertility. Genetics. 1988;118:75-86.

61. Kennedy BK, Austriaco NR Jr, Zhang J, Guarente L. Mutation in the silencing gene SIR4 can delay aging in S. cerevisiae. Cell. 1995;80:485-96.

62. Kenyon C, Chang J, Gensch E, Rudner A, Tabtiang R. A C. elegans mutant that lives twice as long as wild type. Nature. 1993;366:461-4.

63. Hekimi S, Guarente L. Genetics and the specificity of the aging process. Science. 2003;299:1351-4.

64. Ford E, Voit R, Liszt G, Magin C, Grummt I, Guarente L. Mammalian Sir2 homolog SIRT7 is an activator of RNA polymerase I transcription. Genes Dev. 2006;20:1075-80.

65. Gomes AP, Price NL, Ling AJ, Moslehi JJ, Montgomery MK, Rajman $L$, et al. Declining NAD(+) induces a pseudohypoxic state disrupting nuclear-mitochondrial communication during aging. Cell. 2013;155:1624-38.

66. Aquilano K, Vigilanza P, Baldelli S, Pagliei B, Rotilio G, Ciriolo MR. Peroxisome proliferator-activated receptor gamma co-activator 1alpha (PGC1alpha) and sirtuin 1 (SIRT1) reside in mitochondria: possible direct function in mitochondrial biogenesis. J Biol Chem. 2010;285:21590-9.

67. Ghosh S, George S, Roy U, Ramachandran D, Kolthur-Seetharam U. NAD: a master regulator of transcription. Biochim Biophys Acta. 2010;1799:681-93

68. Naimi M, Arous C, Van Obberghen E. Energetic cell sensors: a key to metabolic homeostasis. Trends Endocrinol Metab. 2010;21:75-82.

69. Bordone L, Guarente L. Calorie restriction, SIRT1 and metabolism: understanding longevity. Nat Rev Mol Cell Biol. 2005;6:298-305.
70. Lin SJ, Defossez PA, Guarente L. Requirement of NAD and SIR2 for life-span extension by calorie restriction in Saccharomyces cerevisiae. Science. 2000;289:2126-8.

71. Kaeberlein M, McVey M, Guarente L. The SIR2/3/4 complex and SIR2 alone promote longevity in Saccharomyces cerevisiae by two different mechanisms. Genes Dev. 1999;13:2570-80.

72. Sinclair DA, Guarente L. Extrachromosomal rDNA circles - a cause of aging in yeast. Cell. 1997;91:1033-42.

73. Kohsler M, Leitsch D, Furnkranz U, Duchene M, Aspock H, Walochnik J. Acanthamoeba strains lose their abilities to encyst synchronously upon prolonged axenic culture. Parasitol Res. 2008;102:1069-72.

74. Garajova M, Mrva M, Vaskovicova N, Martinka M, Melicherova J, Valigurova A. Cellulose fibrils formation and organisation of cytoskeleton during encystment are essential for Acanthamoeba cyst wall architecture. Sci Rep. 2019;9:4466.

75. Moon EK, Hong Y, Chung DI, Goo YK, Kong HH. Potential value of cellulose synthesis inhibitors combined with PHMB in the treatment of Acanthamoeba keratitis. Cornea. 2015;34:1593-8.

76. Moon EK, Kong HH. Short-cut pathway to synthesize cellulose of encysting Acanthamoeba. Korean J Parasitol. 2012;50:361-4.

77. Chavez-Munguia B, Omana-Molina M, Gonzalez-Lazaro M, GonzalezRobles A, Bonilla P, Martinez-Palomo A. Ultrastructural study of encystation and excystation in Acanthamoeba castellanii. J Eukaryot Microbiol. 2005;52:153-8.

\section{Publisher's Note}

Springer Nature remains neutral with regard to jurisdictional claims in published maps and institutional affiliations.
Ready to submit your research? Choose BMC and benefit from:

- fast, convenient online submission

- thorough peer review by experienced researchers in your field

- rapid publication on acceptance

- support for research data, including large and complex data types

- gold Open Access which fosters wider collaboration and increased citations

- maximum visibility for your research: over $100 \mathrm{M}$ website views per year

At BMC, research is always in progress.

Learn more biomedcentral.com/submissions 